\title{
LA SOCIEDAD 2.0: DE LA DEMOCRATIZACIÓN DE LOS MEDIOS DE COMUNICACIÓN A LA SOCIALIZACIÓN DE LA PRODUCCIÓN
}

\author{
Society 2.0: from the democratization of media communication \\ to the socialization of production
}

\author{
AUTORES: GÓMEZ ISASSI, José Alberto \\ Profesor Adscrito - Universidad Autónoma de Tamaulipas - Mexico \\ albertomez@gmail.com
}

\begin{abstract}
Resumen
El advenimiento de Internet y con ello las redes sociales puso en marcha una transformación de las formas de organización social sustentada por la web, la telefonía móvil y el acceso ubicuo a la información. En la década entre 2004 y el 2014, la proliferación de herramientas de comunicación hicieron posible la participación en la producción, edición y distribución de información y contenidos, causando una disrupción en los medios de comunicación. Manuel Castells (2010) señala que el consumidor se convierte al mismo tiempo en creador activo con capacidad para contribuir y compartir múltiples visiones del mundo en el que vive. Una fuerte crisis económica y la innovación tecnológica empujó a millones de personas a usar la red y capitalizar su creatividad para crear empresas y servicios a través del emprendimiento y la colaboración colectiva por medio del crowdfunding, y la economía de la colaboración. El aumento de la penetración a internet y los dispositivos móviles conforman una infraestructura compuesta por la interconexión digital de los objetos cotidianos con internet, que está redefiniendo la relación entre las personas, la tecnología y las ciudades. La maduración de una cultura de la participación y el aumento de creadores de contenido abren las vías a una segunda etapa de disrupción digital con el potencial de transformar otras industrias.
\end{abstract}

\section{Palabras clave}

disrupción, emprendimiento, industrias creativas, cultura participativa, economía colaborativa.

\begin{abstract}
The advent of the Internet and online social networks unleashed a transformation of the social structures organized by the web, mobile technologies, and the ubiquitous access to information. During 2004 and 2014, the proliferation of communication tools made possible social participation in the production, editing, and distribution of content and information, disrupting mass communication media. Manuel Castells (2010) argues that the consumer becomes at the same time an active creator capable of contributing and sharing multiple visions of the world in which he inhabits. A huge economic crisis and innovation technology pushed millions of people to rely on the web to capitalize on their creativity to create companies, services and products through entrepreneurship and collective collaboration via crowdfunding and the sharing economy. The rise in Internet adoption and mobile devices form an infrastructure combined by the connection of digital devices with the Internet, redefining the relationship between people, technology and cities. The growth of participatory culture and the rise of content creators are opening new opportunities for a second stage of digital disruption with the potential to transform other industries.
\end{abstract}

\section{Key words}

digital disruption, entrepreneurship, creative industries, participatory culture, sharing economy.

\section{Introducción}

Hace veinte años el internet puso en marcha profundas transformaciones en la comunicación, la economía y la cultura. Durante los primeros diez años cientos de empresas y millones de personas se conectaron a la red, la siguiente década hizo posible que las personas pasaran de consumidores a participantes activos de la producción y distribución de la información. La innovación tecnológica, el acceso a la información, y una nueva generación creativa de millones de personas, han puesto en marcha una nueva ola de cambios que 
hacen posible la socialización y distribución de la producción.

Durante la década de 1994 a 2004, el internet causó la disrupción de la industria de los medios de comunicación - empresas de TV, prensa y radio migraron a la red, - los modelos de producción -cientos de empresas conectadas a través de la red formaron una economía global dinámica en tiempo real, -los hábitos de consumo y transmisión de información, -el abaratamiento de los equipos de cómputo, el aumento del acceso a internet y el volumen de información accesible para las personas -, crearon nuevas oportunidades que impulsaron la participaron colaborativa de millones de personas en la Red.

El acceso a internet, la distribución del conocimiento, y la computación ubicua gracias a la telefonía móvil durante la década de 2004 al 2014, distribuyeron la producción de información entre billones de personas conectadas a una economía creativa global. La crisis mundial del 2008 que sacudió la economía causó altos niveles de desempleo, empujó a las personas en búsqueda de oportunidades de negocio, utilizando herramientas como blogs, redes sociales, streamings, podcasts, videoblogs, etc., para capitalizar y comercializar con su conocimiento y habilidades en condición de freenlance y emprendedores. El poder de los medios de comunicación se democratizó.

El nuevo ecosistema emprendedor que surgió en la década pasada, aceleró la innovación tecnológica, amplió la permeabilidad de la Red, y mejoró las herramientas de producción como impresoras 3D, equipo de laboratorio, máquinas automatizadas, robots, etc. El crecimiento de la participación social en la economía creativa ha puesto en marcha un proceso de socialización y democratización de la producción.

\section{Las Industrias Creativas}

Vinculadas a las tecnologías de información y comunicación, las industrias creativas tienen un papel importante dentro de la economía creativa al servir como válvulas para la circulación de productos y servicios creados por una clase creativa (Florida, 2012). Consecuentemente, no se puede ignorar el hecho de que la innovación tecnológica es un factor determinante en la canalización del output creativo con el input de las economías globales.

En palabras de dos autores referentes en este ámbito, Garnham señala que la expansión de las industrias creativas:

“... ha tenido lugar y solo puede ser entendido y valorado, en el contexto de un debate más amplio sobre el impacto de las tecnologías de la información y la comunicación (TIC) y la digitalización y la relación entre el despliegue de nuevas redes de comunicación y los productos y servicios que ellas transportan” (Garnham, 2011: 31).

\section{La socialización de los medios de comunicación}

El surgimiento y la popularidad de los blogs y las redes sociales a partir 2004 empoderó a millones de personas a crear contenido y compartirlo en Internet, formando rápidamente redes especializadas que im-
Y de manera similar, para Alan Freeman:

Las industrias creativas son un fenómeno económico moderno, un producto de la era de Internet, un sector comercial de la economía de crecimiento rápido en la que el diseño y el dicho 'contenido estético' se encuentran en una relación simbiótica con las habilidades de la información, comunicación, y tecnología o TIC...”(Freeman, 2013).

Freeman articula bajo una lógica mercantil de la estética: que se trata de industrias donde el diseño es la actividad principal, la creatividad humana es el recurso principal y el contenido estético es la fuente principal de demanda. De esta forma, el diseño y la creatividad humana forman parte de la modernidad líquida que hace posible el flujo de contenido e información a través de redes globales sociales y económicas. 
ción de información y contenidos, el consumidor se convierte al mismo tiempo en creador activo con capacidad para contribuir y compartir múltiples visiones del mundo en el que vive" (Castells 2010).

Estas nuevas redes económicas permitieron a miles de personas capitalizar en su expertise, creando empresas y ofreciendo servicios en Red. El internet, las herramientas digitales, y las nuevas plataformas de crecimiento económico y colaboración social crearon oportunidades de generación de ingresos para millones de personas, impulsando el crecimiento de un nuevo ecosistema emprendedor. En un contexto económico, las industrias creativas están vinculadas a una Economía Digital generada por Internet, en que las:

"herramientas tecnológicas se convirtieron en una de las más importantes actividades económicas en la actualidad, al permitir diversificar productos y servicios tales como el software, el hardware y periféricos; que cambiaron los procesos, apoyando a la toma de decisiones modificando las formas del consumo con el comercio electrónico, las transacciones, el entretenimiento" (Alfredo Domínguez y Natalia Pérez: 2013:25).

El impacto social, cultural y económico de la economía creativa es impulsado por una generación de nativos digitales conectada a través de redes globales de información con características homogéneas que tiene una cultura participativa (Jenkins 2006) que consume, comparte, produce, reproduce, y remezcla cualquier contenido digital. Este proceso de socialización y democratización de la información de los medios de comunicación ha hecho posible el surgimiento de una cultura de la participación que es capaz de emprender y crear empresas, ofreciendo productos y servicios, explotando su capital creativo.

\section{Nueva generación de nativos digitales}

Para entender mejor la naturaleza de estas transformaciones sociales es necesario observarlas desde un prisma generacional. La generación milennial, aquellos nacidos entre 1980 y 2000, y la generación Z, nacidos a partir de 2001 representan el primer grupo de personas que ha crecido inmerso en un mundo interconectado a través de la computación ubicua, participando en comunidades dentro de su entorno natural y digital.

Personas de todas las edades y de todos los rincones del planeta utilizan Internet. Sin embargo, como Tapscott (2010) señala; "una nueva generación de jóvenes ha crecido conectada y está introduciendo una nueva ética de la apertura, la participación y la interactividad en los centros de trabajo, las comunidades y los mercados". Esta generación recibe el nombre de «Generación Net» ${ }^{1}$, y está caracterizada por la forma en que trasladan el mismo ethos interactivo que caracteriza a la Web 2.0 (O’Reilly, 2005) y la gran varie-

${ }^{1}$ Expresión acuñada por Don Tapscot en su libro: Growing Up Digital: The rise of the Net Generation, Nueva York, McGraw-Hill, 1997 (trad. Cas.: Creciendo en un entorno digital: la generación Net, Santafé de Bogotá, McGrawHill, 1998) dad de comunidades virtuales dinámicas y redes activas de interacción que sirven como pegamento de sus redes sociales, a ámbitos de la vida cotidiana como el trabajo, la educación y el consumo. O’Reilly sugiere, que la participación de individuos dentro de la Web 2.0 debe ser más una actitud y no precisamente una tecnología. Esta arquitectura de la participación da cuenta de un cambio tecnológico pero más aún de un cambio social que ofrece a las comunidades la posibilidad de contar con herramientas que multipliquen las formas en que se genera y distribuye el conocimiento. Otra característica de los miembros de la generación Net, es la inconformidad que tienen ante el consumo pasivo, ya que cada vez más satisfacen sus deseos de elección, comodidad, personalización y control diseñado, produciendo y distribuyendo ellos mismos los productos. En el libro Economía Digital (1997), Don Tapscott introdujo el término prosumo para describir cómo se está desdibujando la diferencia entre productores y consumidores ${ }^{2}$.

\footnotetext{
${ }^{2}$ Alvin Toffler acuñó por primera vez el término prosumidor
en su obra The Third Wave, Nueva York, Bantam Books,

${ }^{2}$ Alvin Toffler acuñó por primera vez el término prosumidor
en su obra The Third Wave, Nueva York, Bantam Books, (trad. Cast: La tercera ola: la tercera ola está creando nueva civilización, Barcelona, Plaza y Janés, 1990)
} 
Dentro de la generación Net de Tapscott, se encuentran los «nativos»y los «inmigrantes» digitales de Prensky. La generación de jóvenes que ha nacido inmersa en el desarrollo de las nuevas tecnologías, y ha crecido durante las últimas décadas del siglo XX, es la primera generación de «nativos digitales». Se trata de un grupo de personas para las que los juegos de ordenador, Internet, el teléfono celular, el correo electrónico o la mensajería instantánea forman parte integral de sus vidas. Además, como consecuencia de estos usos, la forma de pensar de esta generación ha cambiado y es distinta a la de sus mayores (Prensky, 2001:1).

Una de las características que más ha marcado a esta generación es el aspecto de la participación, concepto que el teórico de los medios Henry Jenkins desarrolla en un contexto de una cultura participativa (Jenkins et al. 2009). Investigaciones sobre el aprendizaje y la cultura participativa han destacado cuatro conjuntos básicos de la práctica dentro de la corriente actual en el espacio mediático digital (Kahne et al. 2014):

Circular: (el uso de blogs, podcasts, o reenviar enlaces)

Colaborar: (mediante el trabajo en conjunto con otros para producir y compartir información a través de proyectos como Wikipedia Crear: (mediante la producción y el intercambio de contenido mediático a través de plataformas como Youtube y Flickr) Conectar: (a través de las redes sociales, como Facebook y Twitter, o a través de comunidades, como clanes de juegos o fandoms).

Lo que hace a la cultura participativa algo único no es la existencia de estos actos individuales; en cambio, el creciente acceso a herramientas que permiten realizar estas cuatro prácticas, y el consecuente aumento de la prevalencia de estos actos está cambiando el contexto cultural en el que las personas operan. Ser parte de una cultura participativa abre nuevas oportunidades y cambia las expectativas de los participantes sobre la forma de abordar una serie de actividades, como el trabajo, el aprendizaje, la política, el consumo, y su participación en el mercado.

\section{Innovación tecnológica}

La innovación tecnológica se entiende como un proceso de simplificación del uso de aparatos electrónicos (hardware) y los programas (software) utilizados en la generación de oportunidades de comercializar con nuevos productos y servicios. Así la innovación se entiende como la introducción de un nuevo, o significativamente mejorado, producto (bien o servicio), de un proceso, de un método de comercialización o de un nuevo método organizativo, en las prácticas internas de la empresa, la organización del lugar de trabajo o las relaciones exteriores.

Según Pavón e Hidalgo (1997), el proceso de innovación tecnológica se define como el conjunto de las etapas técnicas, industriales y comerciales que conducen al lanzamiento con éxito en el mercado de productos manufacturados, o la utilización comercial de nuevos procesos técnicos. El Manual de Oslo, señala que la innovación tecnológica se distingue por una mejora o novedad en las características del desempe- ño de los productos o servicios, y su aplicabilidad en la práctica dependerá del grado en que dichas características y su grado de novedad sean un factor importante en las ventas de una empresa o industria concerniente (De Oslo, 2005).

La innovación tecnológica es el principal disruptor en las industrias que se encuentran ante la digitalización de sus servicios, productos y procesos. La llamada disrupción o innovación disruptiva, es definida por Clayton Christensen como una innovación capaz de crear un mercado nuevo o inesperado mediante el desarrollo de un conjunto diferente de valores. Christensen (2013), señala que esto sucede cuando una tecnología se vuelve 'suficientemente buena' como para substituir una existente a un precio mucho menor, cuando ocurre una migración de una masa crítica que sustenta el uso de esta nueva tecnología, y a su vez hace su uso atractivo para el mercado comercial. 


\section{El ecosistema emprendedor}

Si bien los emprendedores se multiplicaron desde la revolución industrial, la crisis económica del 2008 popularizó e impregnó el concepto en la cultura popular como una alternativa ante el desempleo y el corporativismo. El economista Schumpeter, comenta que los emprendedores son innovadores que buscan destruir el estatus-quo de los productos y servicios existentes para crear nuevos productos y servicios. Asimismo, el experto en administración de empresas Peter Drucker, se refiere al emprendedor como alguien que busca el cambio, responde a él y explota sus oportunidades. La innovación es una herramienta específica de un emprendedor, por ende el emprendedor efectivo convierte una fuente en un recurso.

La globalización del emprendimiento se expandió con base en la aceleración conseguida por un nuevo ecosistema conformado por la popularización de un proceso de investigación de mercado basado en la observación, definición, ideación creativa, prototipado y testeo. Un modelo ágil, que promueve el lanzamiento de productos y servicios en versión Beta, sin un modelo de negocio claro, con una iteración constante, hasta alcanzar la masa crítica de usuarios.

El emprendimiento dentro del sector tecnológico es asociado con un alto nivel de riesgo, razón por la cual es una actividad atractiva para un segmento joven de la sociedad. El emprendimiento ha aumentado en todos los grupos de edad desde 2007. Los mayores aumentos porcentuales se han producido en el grupo de 34-44 años de edad (hasta 2,32 puntos porcentuales), mientras que el mayor porcentaje de los empresarios es en el grupo de 25-34 años de edad (hasta 2,22 puntos porcentuales) (Ács et al. 2015). La posibilidad de crear productos y servicios de desde el hogar ha ido permeando la cultura popular, atrayendo la atención de millones de personas que buscan capitalizar su creatividad para crear un estilo de vida congruente con la cultura participativa de una nueva generación.

\section{La economía de la colaboración}

Así como las tecnologías de información y comunicación hacen posible la colaboración entre personas en la creación de contenido, también permiten la colaboración en el financiamiento de proyectos o negocios de manera colectiva. El financiamiento colectivo (crowdfunding), la colaboración abierta (crowdsourcing), y la economía de la colaboración (sharing economy), son alternativas democráticas para el financiamiento de proyectos e impulsan el crecimiento del ecosistema emprendedor.

El crowdfunding, es un modelo de formación de capital y participación de mercado en donde las necesidades y los propósitos del financiamiento se dan a conocer ampliamente a través de una convocatoria abierta, por lo general, mediante internet, que les permite obtener el apoyo de contribuciones colectivas de diversos donantes o inversionistas independientes (FOMNIN, 2015). Este modelo de microfinanciamiento demuestra un interés de las personas por impulsar una agenda participativa en la producción de los productos que consumen. Algunos de los proyectos más exitosos utilizando crowdfunding, es el smartwatch Pebble, que recaudó $\$ 10.3$ mdd en una campaña que duró un mes durante el 2012, y que buscaba una cantidad inicial de cien mil dólares. El proyecto 'the coolest cooler' recaudó $\$ 13,285,226$ con inversiones de 62,642 personas, y su diseñador utilizó una impresora 3D para crear el prototipo que utilizó para su campaña.

Este tipo de micro-financiamiento evidencia un interés de las personas por impulsar una agenda participativa en la producción de los productos que desean. En un periodo de 2 años se han financiado al menos cinco impresoras 3D; la FORM 1: recaudó $\$ 2,945,885$ usd con una meta inicial de 100,000 usd, The Buccaneer: recaudó 1,438,765 usd de 100,000 usd., RigidBot: $\$ 1,090,098$ de $\$ 31,500$ usd, Printrtbot: $\$ 830,827$ usd de $\$ 25,000$ usd, The Peachy Printer: $\$ 651,091$ usd de $\$ 50,000$ usd (Hensley, 2014). La impresión 3D puede tener un impacto en la industria de la manufactura similar al que tuvieron los blogs y las redes sociales para los medios de comunicación. El semanario Inglés, The Economist, se refirió 
a la impresión 3D, como la 3era Revolución Industrial, después de la mecanización en el siglo XIX, y en ensamblaje en línea del siglo XX.

Las impresión en 3D, encaja con la descripción de una tecnología disruptiva, de acuerdo a Clayton Christensen, quien explica que este tipo de tecnologías continua evolucionando hasta llegar al punto en que pueda satisfacer las necesidades de un mercado de gama alta a un menor costo, momento en el que se adelanta a los actores dominantes. Esta nueva capacidad de participar en el proceso de producción de artículos anteriormente reservados para fabricas, responde a la cultura de la participación de la nueva generación. Una vez democratizado el acceso a herramientas que permiten crear nuevos productos y servicios, las personas pueden organizarse y colaborar utilizando los modelos económicos y de negocio propios de la red.

Algunos países líderes en innovación han puesto en marcha leyes que apoyan la consolidación del crowdfunding, tal es el caso de "Jumpstart Our Business Startups Act" (JOBS Act), firmada por Barack Obama en 2012, que permite fomentar el financiamiento de las pequeñas empresas mediante este esquema.

Las empresas de la economía colaborativa han sido disruptivas en diversas industrias, tal es el caso de Uber con los taxis, Airbnb en la hostelería, BlaBlaCar en el autotransporte, etc. Dentro de la sharing economy las empresas más exitosas reconocen que su mayor valor está en la cultura abierta y participativa que puedan llegar a formar, así como en la generación de confianza entre sus participantes. Este modelo de financiamiento colaborativo es un indicador de la emergencia de cambios sociales mucho más profundos.

\section{La socialización de la producción}

El internet, la innovación tecnológica y la computación ubicua han creado una red de digital que conecta a billones de personas, que hace posible la distribución y circulación de la información, el conocimiento, y la democratización de la producción de contenido creativo. Esta ola de innovación y disrupción en industrias que incluyen la manufactura, medicina, robótica, etc., junto con nuevas herramientas como las impresoras 3-D, las aplicaciones, el crowdfunding, el crowdsourcing, nuevos materiales y más, representan un proceso de socialización y democratización de la producción. De manera que así como pasamos de consumir información a producirla, es posible también que pasemos de consumir productos a producirlos.

El teórico del Internet, Chris Anderson, habla de una 'siguiente revolución industrial' en la que los átomos son los nuevos bits. Esta nueva fase industrial se alimenta de la popularidad que gozan los movimientos como el DIY (Do It Yourself por sus siglas en Inglés) o Maker Movement, hackers de software, etc. El autor y ex-editor de la revista Wired, sugiere que:

"si los últimos 10 años han sido sobre el descubrimiento de los modelos sociales postinstitucionales en la Red, entonces los próximos diez años serán sobre a la aplicación de estos en el mundo físico" (Anderson, 2010).

De acuerdo con Anderson, los cambios transformacionales suceden cuando las industrias se democratizan, cuando son arrebatadas del dominio exclusivo de las empresas, gobiernos, y otras instituciones, y entregadas al ciudadano común. El internet democratizó las publicaciones, las transmisiones, y la comunicación, y tuvo consecuencias masivas. La democratización de la producción, distribución y comercialización de productos físicos, sumado al avance exponencial de la innovación tecnológica, hace probable consecuencias igualmente masivas.

Aunque esta nueva revolución industrial parece ofrecer grandes oportunidades para la innovación y el crecimiento económico, es cierto que los países al frente de esta revolución creadora cuentan con un capital humano altamente especializado en ciencias exactas: Ciencia, Tecnología, Ingeniería, y Matemáticas (STEM por sus siglas en inglés).

Este proceso de democratización y socialización de la producción, sucede en un contexto tecnológica que incluye nuevas disciplinas y áreas de oportunidad como el big data, internet de las cosas, inteligencia artificial, robótica, nanotecnología, biotecnología, 
etc. La proliferación de dispositivos móviles, la computación ubicua, y el enorme volumen de datos generado cada día-, es un claro indicador de la necesidad de apoyar al ecosistema emprendedor e impulsar las STEM para buscar el desarrollo social y económico de las sociedad.

\section{Una nueva infraestructura digital de las cosas}

A medida que la penetración de internet y el número de dispositivos contactados a la Web aumentan, ingenieros y diseñadores se enfocan en crear nuevos productos y servicios que mejoren la experiencia de las personas al interactuar con la tecnología. Este enfoque de resolución de problemas, diseño e iteración, acelera la innovación tecnológica.

El ecosistema emprendedor adopta la sistematización de un pensamiento orientado al perfeccionamiento de servicios y productos a través de un proceso creativo. De acuerdo con Wikipedia, Design Thinking se refiere a los métodos y procesos para investigar problemas que no están claramente definidos, obteniendo información, analizando contenido y proponiendo soluciones en los campos del diseño y la planificación. El Design Thinking se presenta como una forma de pensar en la que se combina el conocimiento y compresión del contexto en el que surgen los problemas, la creatividad para presentar propuestas novedosas de solución y la racionalidad para confrontar dichas soluciones creativas con los límites que la realidad impone.

La asimilación de un proceso creativo y constructivo por la generación de nativos digitales acelera la innovación tecnológica, buscando crear su propia realidad a partir de una nueva cultura participativa. Sumado a esto, la proliferación de los dispositivos que se pueden conectar a la red, conforman una infraestructura compuesta por la interconexión digital de objetos cotidianos con internet, un fenómeno que ha sido llamado el internet de las cosas (IoT, por sus siglas en inglés). Los dispositivos también se han adherido al cuerpo de las personas por medio de la tecnología portable (wearables). También se ha despertado una creciente obsesión de medir cada actividad de la vida diaria de las personas, buscando optimizar y maximizar el rendimiento del tiempo, y popularizando tendencias como el "Yo Cuantificable" (quantified self).
Así, la multiplicación de la generación de datos crea grandes oportunidades para empresas de telecomunicaciones como Cisco, quién publicó un reporte en donde señala que el Internet de las Cosas nació oficialmente entre 2008-2009. Este fue el punto en el que el número de dispositivos superó el número de personas en la tierra. En el 2014 el número de aparatos conectados superó el número de personas conectadas a internet. En el 2015 la población alcanzó los 7.2 billones de personas y el número de dispositivos conectados haciende a 25 billones. Para en el 2025, se estima que habrá 50 billones de dispositivos conectados (Evans, 2011).

Toda esta conectividad ha incrementado la cantidad de datos generados, los cuales han intentado ser cuantificados por la empresa de analíticas Domo (James, 2014), dando como resultado un estudio en el que se muestra lo que sucede durante un minuto en Internet; en Facebook se generan 2.5 millones de piezas de contenido compartidas, Twitter cerca de 300,000 mensajes, Instagram 220,000 nuevas fotos, YouTube se suben 72 horas de nuevos videos, Apple 48,000 descargas de Apps, Email 200 millones de mensajes., Amazon genera más de $\$ 80,000$ en ventas online, Google: 4,000,000 millones de búsquedas, Whatsapp 347,222 fotos. Este hecho inmediatamente sugiere algunas preguntas, ¿qué pasa con todos estos datos?, ¿quién se queda con todos estos datos?, y no menos importante, ¿con qué motivo y para qué? El comediante John Oliver nos recuerda en uno de sus programas que no formularnos estas preguntas es como dejar un bebe con un perro salvaje de niñera.

Consecuentemente, todos estos datos han pavimentado el camino hacia el surgimiento de las ciudades inteligentes, lugares donde las tecnologías de la información se combina con la infraestructura, arquitectura, objetos de la vida diaria, incluso en nuestros cuerpos para atender problemas sociales, económicos y hasta medio ambientales (Townsend, 2013). 
El advenimiento de la tecnología móvil y la conectividad ubicua nos lleva hacia el rumbo de las ciudades inteligentes, y en el volante están corporaciones como: IBM, General Electrics, Siemens, y Cisco. Estas empresas están tejiendo un paquete de nuevas tecnologías como el internet de las cosas, los análisis predictivos, y el big data - promoviéndolo con una gran campaña de marketing-, que busca integrarlas a la ciudades.

La infraestructura de las ciudades inteligentes está siendo construida de dos diferentes ángulos. El primero es de arriba hacia abajo, una implementación jerárquica impuesta por grandes empresas que buscan conectar a las ciudades y las personas. Sin embargo, como señalamos arriba, es importante saber que se hace con estos datos, un ejemplo es el proyecto Urbanomics Mobility Project, entre Mastercard- que procesa 43 billones transacciones (por año), en asociación con Cubic, una empresa tecnológica de análisis y visualización de datos acerca del transporte - para obtener información sobre la forma en que el transito y la actividad económica se relacionan en las ciudades. Sobre esto, el vicepresidente ejecutivo y director de servicios para soluciones de gobierno en MasterCard, Ed Brand, declaró que: "reconocemos que una de nuestras grandes áreas de oportunidad es como podemos ayudar al gobierno a tomar decisiones. Debido a la rápida urbanización, debemos contar con una infraestructura eficiente. Nosotros podemos ayudar. Nosotros brindamos al gobierno con la información sobre en donde sucede la actividad económica por estado, región y mercado. Ellos encuentran que nuestra información es mucho más rápida que la de ellos" (Cubic, 2015).

Usar los datos generados en la ciudad dentro de la planeación urbana se está convirtiendo en un nuevo paradigma. Pero al seguir ciegamente los datos, ¿estamos perdiendo de vista el lado humano de las ciudadanos? El periodista británico Steven Poole (2014), en un artículo reciente propone algunas interrogantes como ¿cuál será el rol de las personas? ¿acaso el de un trabajador sin salario, contribuyendo con información voluntariamente a un centro de datos urbanos que es monetizado por compañías privadas?

Si las corporaciones están brindando al gobierno la información para tomar "mejores decisiones". Podemos pensar que hay un interés económico del lado de la empresa. Luego entonces, las corporaciones están moldeando las decisiones que toma el gobierno para construir las "ciudades inteligentes", por lo que cabría preguntarnos, ¿inteligentes para quién?

\section{La innovación abierta}

Las ciudades inteligentes también surgen de forma orgánica por medio de la participación activa, y creativa, de los mismo ciudadanos. Este segundo enfoque de las ciudades inteligentes, responde a la cultura participativa de la generación de nativos digitales, los milenials, han sido nombrados persona del año por la revista TIME en dos ocasiones, la primera en el 2006, por ser los que controlan la Edad de la Información, y en el 2011 por convertirse en el "El Manifestante", por su participación y activismo durante la ola de inconformidad y activismo político que surgió en el medio oriente con las revoluciones árabes.

La contraparte de la tendencia a integrar la tecnología a las ciudades de un modelo de arriba hacia abajo, es la innovación abierta (open source innovation). Este tipo de innovación abierta es impulsada a través de la apro- apropiación de la tecnología de consumo comercial, el código abierto, y la aplicación de los cuatro conjuntos básicos de la práctica dentro de la corriente actual en el espacio mediático digital: circular, colaborar, crear y conectar. En Kibera, un asentamiento informal en los suburbios de Nairobi, África, reclutaron a las personas jóvenes que estuvieran activas en su comunidad, en dos días de entrenamiento sobre cómo usar recibidores de GPS, estos jóvenes cartógrafos voluntarios eran enviados a recorrer Kibera a pie, usando sus cuerpos como herramientas para recolectar miles de calles, callejones y caminos que formarían el primer mapa digital de esta comunidad emergente. Hicieron el mapa sólo en tres semanas. La aplicación de las tecnologías en este tipo de situaciones, se apoya de herramientas de código abierto como OpenStreetMap (OSM), un proyecto colabora- 
tivo para crear un mapa gratuito del mundo. Es considerado un ejemplo exitoso de información geográfica creada por voluntarios. Esta App fue crucial durante el terremoto del 2010 en Haití, que destruyó la agencia nacional de mapeo, además contribuyó con datos esenciales para los esfuerzos de rescate de organizaciones. Otra herramienta comúnmente utilizada es Ushahidi, quiere decir "testimonio" en Swahili, que permite enviar reportes vía msg, móvil, email, pc, y que fue desarrollada para ubicar reportes de violencia en Kenya después de los periodos violentos durante las elecciones en el 2008. Desde entonces, miles han utilizado esta tecnología creada bajo la filosofía crowdsourcing para alzar su voz no sólo en África, pero también en todo el mundo, durante el derrame de petróleo en el Golfo de México se creo un mapa que ubicaba animales atrapados en la costa norteamericana, también durante las elecciones presidenciales del 2012 en México, fue utilizada por activistas para supervisar y reportar incidentes en las casillas de votación.

\section{Conclusión}

Estamos ante la emergencia de una nueva generación conectada, participativa, marcada por la innovación tecnológica, motivada por la posibilidad de utilizar su ventaja competitiva, el saber tecnológico, para transformar su entorno, y que además está equipada con nuevas herramientas que les permite interactuar con el mundo virtual y físico de manera simultánea.

Por un lado estamos ante el derrumbe de una industria construida durante el siglo XX, impuesta por los medios de comunicación masivos, que formó a una sociedad con estructuras jerárquicas, de audiencias muchas veces pasivas - de consumidores. La industria de los medios de comunicación ha sido victima de procesos disruptivos causados por las TIC, y muy a pesar de utilizar artimañas legales, económicas y políticas, finalmente terminaron cediendo ante la distribución y la democratización de la producción de información. Startups que se convirtieron en empresas como Netflix, Buzzfeed, Vice, The Huffington Post, etc., desplazaron en menos de diez años a empresas con más de cien. Sin embargo, en primer lugar la innovación tecnológica debe tomear en cuenta lo mucho por hacer para cerrar la brecha digital y au- mentar la inclusión digital; segundo, esta misma disrupción ha entregado un gran poder a las nuevas corporaciones dueñas de la información que las personas producen, poniendo en riego las libertades fundamentales del ser humano, como es el derecho a la privacidad.

Por otro lado, una nueva generación nacida en la Era de Internet, que creció con las redes sociales, la telefonía móvil, la computación ubicua, y que ha adquirido una nueva cultura participativa que destaca la circulación, colaboración, creación y conexión entre iguales, ha adoptado como estilo de vida la resolución de retos y problemas de forma creativa. Los nuevos emprendedores han comenzado una cruzada contra las viejas estructuras corporativas surgidas de la industria de los medios masivos de comunicación, para imponer una nueva industria del emprendimiento haciendo uso del saber tecnológico adquirido durante toda su vida, la generación de nativos digitales busca imponer un nuevo orden mundial con base en la innovación tecnológica, la disrupción digital y la optimización de la vida.

\section{Referencias}

- $\quad$ Ács, Z. J., Szerb, L., \& Autio, E. (2015). 2016 Global entrepreneurship and development index 2011. Global Entrepreneurship Development Institute. Consultado el 11 de Noviembre de 2015, de http: / / thegedi.org/2016-global-entrepreneurshipindex/
- Anderson, C. (2010). In the Next Industrial Revolution, Atoms Are the New Bits. Consultado el 4 de Agosto del, 2015, en http: / / www.wired.com/2010/01/ff_newrevolution /

- Carriles, L. (2015). Inadem, del subejercicio a la opacidad. Consultado el 21 August 2015, en Eleconomista.com.mx: 
http: / / eleconomista.com.mx/industrias/2014/12/15/ina dem-subejercicio-opacidad

- Castells, M. (2010): Comunicación y poder. Madrid: Alianza.

- Christensen, C. (2013). The innovator's dilemma: when new technologies cause great firms to fail. Harvard Business Review Press.

- MasterCard Social Newsroom,. (2015).Cubic and MasterCard Launch the Urbanomics Mobility Project. Consultado 12 Noviembre 2015, de http: / / newsroom.mastercard.com/pressreleases/cubic-and-mastercard-launch-theurbanomics-mobility-project/

- De Oslo, M. (2005). Guía para la recogida e interpretación de datos sobre innovación. Luxembourg: OECD.

- Domínguez, A., Pérez N. en Godínez R. M., Ortiz J. L. (Cord.) (2013). Economía y Cultura. México. UACM.

- Evans, D. (2011). The internet of things: How the next evolution of the internet is changing everything. CISCO white paper.

- Florida, R. (2002). The creative class. The rise of the creative class.

- $\quad$ FOMIN (2015). Primer estudio sobre crowdfunding realizado en México posiciona al país como líder en América Latina. Consultado el 15 Septiembre 2015, en http: / / www.fomin.org/es /PORTADA / Noticias / Comuni cadosdeprensa / ArtMID /3819/ArticleID / $1100 /$ Primerestudio-sobre-crowdfunding-realizado-en-

M\%C3\%A9xico-posiciona-al-pa\%C3\%ADs-como-

1\%C3\%ADder-en-Am\%C3\%A9rica-Latina.asp

- Freeman, A. (2013). Invertir en la Civilización: presentación sobre las industrias creativas, Mexico. Discurso. Consultado el 22 de Agosto de 2015, en Adademica.edu:

https: / /www.academia.edu/3625913/Invertir_en_la_ Civiliza-

cion_presentacion_sobre_las_industrias_creativas_Mexic o_in_Spanish

- Garnham, N. (2011). Industrias Creativas: Amenazas sobre la cultura digital. España.Gedisa.

- Global Entrepreneurship Development Institute (2015) Global Entrepreneur Index. Consultado el 20 de
Agosto del, 2015, en http://thegedi.org/2015-globalentrepreneurship-index /

- Hensley, M. (2014). 5 Most Successful 3D Printer Crowdfunding Projects of All-Time. 3DPrint.com. Consultado el 10 de Noviembre 2015, en http: / /3dprint.com/294/5-most-successful-3d-printercrowdfunding-projects-of-all-time/

- Hidalgo, P. J., \& Pavón, J. (1997). Gestión e Innovación. Un enfoque estratégico.

- James, J. (2014). Data Never Sleeps 2.0. (2014-0423) [2014-07-20]. http://www. domo. co$\mathrm{m} /$ blog/2014/04/datanever-sleeps-2-0.

- Kahne, J., Middaugh, E., \& Allen, D. (2014). Youth, new media, and the rise of participatory politics.

- McCarthy, N. (2015). The Countries With The Most Engineering Graduates. Forbes. Consultado el 21 de Agosto 2015, de http://www.forbes.com/sites/niallmccarthy/201 5/06/09/the-countries-with-the-mostengineering-graduates-infographic/

- Ojeda, M. (2015). Ajustan reglas del Inadem ante recorte de presupuesto. Consultado el 21 Agosto 2015, en Elfinanciero.com.mx: http://www.elfinanciero.com.mx/economia/inad em-recibira-mil-188-mdp-de-presupuesto-para2015.html

- O'Reilly, T. (2005). What is Web 2.0? Design Patterns and Business Models for the Next Generation of Software. O’Reilly Network. Recuperado de http://www.oreillynet.com/pub/a/oreilly/tim/n ews/2005/09/30/what-is-web-20.html

- Presnky, M. (2001). Digital natives, digital immigrants. On the Horizon, MCB University Press, 9.

- Tapscott, D., Williams, A.D. (2007). Wikinomics La nueva economía de las multitudes inteligentes. Madrid: Paidós

- Townsend, A. M. (2013). Smart cities: big data, civic hackers, and the quest for a new utopia. WW Norton \& Company.

- Wikipedia,. (2015). Design thinking. Consultado el 10 de Noviembre de 2015, en https: / / en.wikipedia.org/wiki / Design_thinking

\section{Forma de Citación}

GÓMEZ ISASSI, José Alberto: La Sociedad 2.0: De la democratización de los medios de comunicación a la socialización de la producción. Revista Communication Papers, No 8 (Monográfico II), páginas 21 a 30 . Departamento de Filología y Comunicación de la Universidad de Girona. Recuperado el _ de__ de $2 \ldots$ de $\underline{\text { http: //www.communicationpapers.es }}$ 